\title{
Piecewise constant bounds for the solution of nonlinear Volterra-Fredholm integral equations
}

\author{
S. YAZDANI and M. HADIZADEH* \\ Department of Mathematics, K.N. Toosi University of Technology, \\ P.O. Box 16315-1618, Tehran, Iran \\ E-mails: yazdanis@sina.kntu.ac.ir / hadizadeh@kntu.ac.ir
}

\begin{abstract}
In this paper, we compute piecewise constant bounds on the solution of mixed nonlinear Volterra-Fredholm integral equations. The enclosures are in the form of intervals which are guaranteed to contain the exact solution considering all round-off and truncation errors, so the width of interval solutions allows us to control the error estimation. An iterative algorithm to improve the accuracy of initial enclosures is given and its convergence are also investigated. Our numerical experiments show that the precision of interval solutions are reasonable in comparison to the classical methods and the obtained conditions and initial enclosure of the proposed algorithm are not restrictive.
\end{abstract}

Mathematical subject classification: $65 \mathrm{G} 20,45 \mathrm{G} 10,65 \mathrm{G} 40$.

Key words: Volterra-Fredholm integral equations, enclosure methods, interval analysis, guaranteed error bounds.

\section{Introduction}

During the last three decades, the role of enclosure methods in numerical solution of operator equations has continuously increased. In many cases computers are not perfect tools in scientific calculations, specially when using floating point arithmetic, the solutions are affected by rounding errors and this may led to completely wrong results (see e.g. [17]). This is more important when increasing

\#CAM-370/11. Received: 12/V/11. Accepted: 02/I/12.

*Corresponding author. 
the speed of computers and for complicated equations, but its impossible to verify the accuracy of the results by classical schemes. Interval enclosure methods are able to compute guaranteed error bounds including all discretization and roundoff errors in the computation. These methods are used in many applied areas e.g. fuzzy set theory, engineering problems with interval valued parameters or interval initial values, robust control, robotics, multimedia architectures [12], economics, beam physics, global optimization, signal processing and computer graphics [11] (see also [1] for a survey on enclosure methods).

Here, we restrict our attention to the computation of guaranteed bounds for the solution of the following nonlinear Volterra-Fredholm integral equation of mixed type

$$
\begin{aligned}
u(x, t)= & f(x, t)+\int_{0}^{t} \int_{\Omega} k(x, t, \xi, \tau, u(\xi, \tau)) d \xi d \tau, \\
& (x, t) \in[0, \chi] \times \Omega,
\end{aligned}
$$

where $u(x, t)$ is an unknown function, and $f(x, t)$ and $k(x, t, \xi, \tau, u(\xi, \tau))$ are known analytic functions defined on

$$
D:=[0, \chi] \times \Omega \quad \text { and } S \times \mathbb{R}
$$

(where $S:=\{(x, t, \xi, \tau): 0 \leq \tau \leq t \leq \chi ;(x, \xi) \in \Omega \times \Omega\})$, with $k(x, t$, $\xi, \tau, u)$ nonlinear in $u$ and $\Omega$ is closed subset of $\mathbb{R}^{d}(d=1,2,3)$.

These equations play an important role for abstract formulation of many initial or boundary value problems of perturbed differential equations, nonlinear parabolic partial differential equations and partial integro-differential equations which arise in various applications like the mathematical modeling of chemical reaction kinetics, population dynamics [25], heat-flow in material with memory, electromagnetics [10], viscoelastic and reaction diffusion problems.

There has been much work on analyzing and numerical methods for the models consisting of Volterra-Fredholm integral equations. Diekmann and Thieme described and developed some of these models in [7, 24]. The existence and uniqueness of the global solution of the equations have been investigated by Burton [4] and Dhakne et al. [6]. Also some of the schemes which have considered the solution of the linear and nonlinear cases of (1.1) numerically are projection methods, continuous time and discrete time spline collocation method 
$[2,16]$, Euler Nystrom and trapezoidal Nystrom methods [13], the Adomian decomposition method [18], the time-stepping methods by a certain choice of direct quadrature (DQ) [3] and Sinc collocation method based on DE transformation in [14]. Generally, all of these classical methods are efficient and effective, but in many cases most of them are unable to control the truncation and round off errors in numerical computations. Here, our aim is to use an interval scheme which considers such these apparent errors.

As we know, there are not any considerable interval based works in solving mixed Volterra-Fredholm integral equations. Moore [19] has introduced an iterative algorithm for nonlinear integral equations based on the definition of interval integration. Caprani et al. [5] and Dobner [9] proposed several papers on solving Fredholm integral equations using enclosure methods. Recently, Murashige and Oishi [21] presented numerical verification of solutions of periodic integral equations with a singular kernel and Nekrasov's integral equation. In comparison to Fredholm type, there are a few enclosure methods on solving Volterra integral equations especially in the nonlinear form $[8,15]$.

In the present work, we propose an algorithm to provide piecewise constant bounds for the solution of nonlinear Volterra-Fredholm integral equations. Our algorithm is based on the early idea of Moore [19] for Volterra integral equations. The organization of this paper is as follows: In section 2, we give basic notation, definitions and a summary on the principles of interval arithmetic. A new enclosure is constructed from an initial bound and its properties are studied in section 3 and then an iterative algorithm for the computation of piecewise constant bounds including its convergence conditions is presented. Finally, numerical experiments and a full discussion on initial enclosure, the algorithm conditions and also the number of iterations are reported to clarify the efficiency of the method.

\section{Notations and auxiliary results of the interval theory}

\subsection{Interval arithmetic}

We first introduce some basic properties of interval arithmetic from [20, 22, 23]. The set of compact real intervals is denoted by

$$
\mathbb{I} \mathbb{R}=\{\mathbf{x}=[\underline{x}, \bar{x}] \mid \underline{x}, \bar{x} \in \mathbb{R}, \underline{x} \leq \bar{x}\} .
$$


Through out of this paper, using the notation of [23], intervals are denoted by boldface and lowercase letters are used for denoting scalars and vectors. A real number $x$ is identified with a point interval $\mathbf{x}=[x, x]$. The quality of interval analysis is measured by the width of the interval results, and a sharp enclosure for the exact solution is desirable. The midpoint and the width of an interval $\mathbf{x}$ are denoted by

$$
m(\mathbf{x}):=(\underline{x}+\bar{x}) / 2 \text { and } \quad w(\mathbf{x}):=\bar{x}-\underline{x},
$$

respectively. The width of an interval vector $\mathbf{x}=\left(\mathbf{x}_{1}, \ldots, \mathbf{x}_{n}\right)$ is the largest of the widths of any of its component intervals

$$
w(\mathbf{x})=\max w\left(\mathbf{x}_{i}\right), \quad i=1, \ldots, n .
$$

Considering $|\mathbf{x}|=\max \{|\underline{x}|,|\bar{x}|\}$, for any $\mathbf{x}, \mathbf{y} \in \mathbb{I} \mathbb{R}$ and $a, b \in \mathbb{R}$, we get the following properties:

$$
\begin{gathered}
w(a \mathbf{x}+b \mathbf{y})=|a| w(\mathbf{x})+|b| w(\mathbf{y}), \\
w(\mathbf{x y}) \leq|\mathbf{x}| w(\mathbf{y})+|\mathbf{y}| w(\mathbf{x}) .
\end{gathered}
$$

The four elementary operations of real arithmetic can be extended to intervals. Operations over intervals $\diamond \in\{+,-, *, /\}$ are defined by the general rule

$$
\mathbf{x} \diamond \mathbf{y}=\{x \diamond y \mid x \in \mathbf{x}, y \in \mathbf{y}\} .
$$

It is easy to see that the set of all possible results when applying an operator $\diamond$ to $\mathbf{x}$ and $\mathbf{y}$, forms a closed interval (for $0 \notin \mathbf{y}$ in the case of division) and the end point can be calculated by

$$
\mathbf{x} \diamond \mathbf{y}=\{\min (x \diamond y), \max (x \diamond y)\}, \quad \text { for } \quad \diamond \in\{+,-, *, /\} .
$$

The following lemma gives an important property which is used frequently in this paper:

Lemma 1. Let $\mathbf{k}=[\underline{k}, \bar{k}]$ and $\mathbf{y}=[0, \bar{y}]$, also there exists a constant $\alpha>1$, such that $|\mathbf{k}| \leq \alpha w(\mathbf{k})$, then

$$
w(\mathbf{k y}) \leq \alpha w(\mathbf{k}) \bar{y}
$$


Proof. We consider the following three cases:

Case 1. If $\underline{k} \leq 0 \leq \bar{k}$, then $w(\mathbf{k y})=w([\underline{k} \bar{y}, \bar{k} \bar{y}])=\bar{y}(\bar{k}-\underline{k}) \leq \alpha w(\mathbf{k}) \bar{y}$.

Case 2. If $\underline{k} \leq \bar{k} \leq 0$, then $w(\mathbf{k y})=w([k \bar{y}, 0])=|\underline{k}| \bar{y} \leq|\mathbf{k}| \bar{y} \leq \alpha w(\mathbf{k}) \bar{y}$.

Case 3. If $0 \leq \underline{k} \leq \bar{k}$, then $w(\mathbf{k y})=w([0, \bar{k} \bar{y}])=|\bar{k}| \bar{y} \leq|\mathbf{k}| \bar{y} \leq \alpha w(\mathbf{k}) \bar{y}$, and the proof is complete.

\subsection{Preliminaries}

Most of the following information regarding the interval functions may be found in [20], but here we recall certain points that are important for establishing and analyzing the accuracy of our new enclosures from [23] and [19]:

Let $f$ be a real valued function of several variables, we can define set image or the united extension of $f$ as:

$$
f\left(\mathbf{x}_{1}, \ldots, \mathbf{x}_{n}\right)=\left\{f\left(x_{1}, \ldots, x_{n}\right): x_{1} \in \mathbf{x}_{1}, \ldots, x_{n} \in \mathbf{x}_{n}\right\} .
$$

Functions of interval variables are often computed by substituting the given interval $\mathbf{x}$ for $x$ in $f(x)$ and then evaluating the function using interval arithmetic. An interval extension of $f$ is an interval valued function $F$ of $n$ interval variables $\mathbf{x}_{1}, \ldots, \mathbf{x}_{n}$ such that for real arguments $x_{1}, \ldots, x_{n}$ we have

$$
F\left(\mathbf{x}_{1}, \ldots, \mathbf{x}_{n}\right)=f\left(x_{1}, \ldots, x_{n}\right) .
$$

This natural interval extension is sometimes wider than the actual range of function values, though it always includes the actual range e.g. let $f(x)=$ $x /(1+x)$, then $f([1,2])=[1 / 2,2 / 3]$, but natural interval extension of $f$ is $F([1,2])=[1 / 3,1]$ which is wider than $[1 / 2,2 / 3]$. To overcome this difficulty so called dependency effect, some alternative schemes such as mean value form and Taylor enclosures are proposed in [20] and [5].

Definition 1. [20] An interval extension $F$ is said to be Lipschitz interval extension in $\mathbf{x}_{0}$, if there is a constant $L$, such that $w(F(\mathbf{x})) \leq L w(\mathbf{x})$, for every $\mathbf{x} \subseteq \mathbf{x}_{0}$.

Therefore, the width of $F(\mathbf{x})$ approaches zero at least linearly with the width of $\mathbf{x}$. Here $\mathbf{x}$ may be an interval or an interval vector. 
Definition 2. [20] An interval valued function $F\left(\mathbf{x}_{1}, \ldots, \mathbf{x}_{n}\right)$ is called inclusion isotonic, if for $\mathbf{y}_{i} \subseteq \mathbf{x}_{i}, \quad i=1, \ldots, n$ we have

$$
F\left(\mathbf{y}_{1}, \ldots, \mathbf{y}_{n}\right) \subseteq F\left(\mathbf{x}_{1}, \ldots, \mathbf{x}_{n}\right) .
$$

Definition 3. [20] A rational interval function is an interval-valued function whose values are defined by a specific finite sequence of interval arithmetic operations.

Lemma 2. [20] If $F$ is a natural interval extension of a real rational function with $F(\mathbf{x})$ defined for $\mathbf{x} \subseteq \mathbf{x}_{0}$, where $\mathbf{x}$ and $\mathbf{x}_{0}$ are intervals or n-dimensional interval vectors, then $F$ is Lipschitz in $\mathbf{x}_{0}$.

Lemma 3. [20] Let $F$ and $G$ be inclusion isotonic interval extensions with $F$ Lipschitz in $Y_{0}, G$ Lipschitz in $\mathbf{x}_{0}$, and $G\left(\mathbf{x}_{0}\right) \subseteq Y_{0}$, then the composition $H(\mathbf{x})=F(G(\mathbf{x}))$ is Lipschitz in $\mathbf{x}_{0}$ and be inclusion isotonic.

Lemma 4. [20] If $F$ is a rational interval extension of a real function $f$, and $\left(x_{1}, \ldots, x_{n}\right) \in\left(\mathbf{x}_{1}, \ldots, \mathbf{x}_{n}\right)$ then $f\left(x_{1}, \ldots, x_{n}\right) \in F\left(\mathbf{x}_{1}, \ldots, \mathbf{x}_{n}\right)$.

A well known definition of the interval extension of a real integral has been introduced by Moore [19] which is defined as follows:

$$
\int_{a}^{x} f(x) d x \in \int_{[a, x]} f(x) d x=F([a, x])(x-a),
$$

where $F$ is interval extension of $f$.

\section{Computation of piecewise constant bounds}

In order to compute piecewise constant bounds for the solution of a nonlinear Volterra-Fredholm integral equation (1.1), we first subdivide the intervals $[0, \chi]$ and $\Omega=[a, b]$ by points

$$
\begin{gathered}
0=x_{0}<x_{1}<\cdots<x_{p_{x}}=\chi, \quad \mathbf{x}_{i}=\left[x_{i-1}, x_{i}\right], i=1, \ldots, p_{x}, \\
a=t_{0}<t_{1}<\cdots<t_{p_{t}}=b, \quad \mathbf{t}_{j}=\left[t_{j-1}, t_{j}\right], j=1, \ldots, p_{t},
\end{gathered}
$$

where $p_{x}$ and $p_{t}$ are number of subdivisions. Now, considering an initial enclosure $\mathbf{u}_{0}=\left[\underline{u_{0}}, \overline{u_{0}}\right]$, which is assumed to contain the exact solution of the equation in whole interval, a new enclosure in each subinterval will be obtained. 
Suppose that the real valued function $f(x, t)$ in (1.1) is continuous and $k(x, t$, $\xi, \tau, u(\xi, \tau))$ satisfies a Lipschitz condition with respect to $u$ for all $(x, t, \xi$, $\tau, u(\xi, \tau))$ in $S \times \mathbb{R}$, also $F\left(\mathbf{x}_{i}, \mathbf{t}_{j}\right)$ and $\left.K\left(x, t, \xi, \tau, \mathbf{u}_{0}\right)\right)$ are natural interval extensions of $f$ and $k$. Hence using Lemmas 2 and 3 and considering $L_{F}$ as a Lipschitz constant, we conclude $F\left(\mathbf{x}_{i}, \mathbf{t}_{j}\right)$ is Lipschitz in $[0, \chi] \times \Omega$, so

$$
w\left(F\left(\mathbf{x}_{i}, \mathbf{t}_{j}\right)\right) \leq L_{F} L_{\delta},
$$

where $L_{\delta}=\max \left\{w\left(\mathbf{x}_{i}\right), w\left(\mathbf{t}_{j}\right)\right\}$. Also, there exist a Lipschitz constant $L_{k}$ such that for all $x, \xi \in[0, \chi]$, and $t, \tau \in \Omega$, we have

$$
w\left(K\left(x, t, \xi, \tau, \mathbf{u}_{0}\right)\right) \leq L_{k} w\left(\mathbf{u}_{0}\right) .
$$

In the following, we introduce the new enclosure and give some of its important properties:

Lemma 5. Let $u(x, t) \in \mathbf{u}_{0}=\left[\underline{u_{0}}, \overline{u_{0}}\right]$ and for $x \in \mathbf{x}_{i}=\left[x_{i-1}, x_{i}\right], \xi \in$ $\mathbf{x}_{l}, 1 \leq l \leq i \leq p_{x}$, and $t \in \mathbf{t}_{j}=\left[t_{j-1}, t_{j}\right], \tau \in \mathbf{t}_{k}, 1 \leq k \leq j \leq p_{t}$, we have $f(x, t) \in F\left(\mathbf{x}_{i}, \mathbf{t}_{j}\right), k(x, t, \xi, \tau, u(x, t)) \in K\left(\mathbf{x}_{i}, \mathbf{t}_{j}, \mathbf{x}_{l}, \mathbf{t}_{k}, \mathbf{u}_{0}\right)$, then we can obtain a new enclosure for each $\left.u(x, t)\right|_{x \in \mathbf{x}_{i}, t \in \mathbf{t}_{j}}$ as

$$
\begin{aligned}
& \left.u(x, t)\right|_{x \in \mathbf{x}_{i}, t \in \mathbf{t}_{j}} \in \mathbf{u}_{1, i, j}:=F\left(\mathbf{x}_{i}, \mathbf{t}_{j}\right) \\
& +\sum_{k=1}^{j-1} \sum_{l=1}^{p_{x}} K\left(\mathbf{x}_{i}, \mathbf{t}_{j}, \mathbf{x}_{l}, \mathbf{t}_{k}, \mathbf{u}_{0}\right) w\left(\mathbf{x}_{l}\right) w\left(\mathbf{t}_{k}\right) \\
& +\sum_{l=1}^{p_{x}} K\left(\mathbf{x}_{i}, \mathbf{t}_{j}, \mathbf{x}_{l}, \mathbf{t}_{j}, \mathbf{u}_{0}\right) w\left(\mathbf{x}_{l}\right)\left[0, w\left(\mathbf{t}_{j}\right)\right]
\end{aligned}
$$

Proof. Let us assume $x \in \mathbf{x}_{i}$ and $t \in \mathbf{t}_{j}$ in each subinterval, then the equation (1.1) can be written as

$$
\begin{aligned}
u(x, t) & =f(x, t)+\int_{0}^{t} \int_{\Omega} k(x, t, \xi, \tau, u(\xi, \tau)) d \xi d \tau \\
& =f(x, t)+\sum_{k=1}^{j-1} \sum_{l=1}^{p_{x}} \int_{t_{k-1}}^{t_{k}} \int_{x_{l-1}}^{x_{l}} k(x, t, \xi, \tau, u(\xi, \tau)) d \xi d \tau \\
& +\sum_{l=1}^{p_{x}} \int_{t_{j-1}}^{t} \int_{x_{l-1}}^{x_{l}} k(x, t, \xi, \tau, u(\xi, \tau)) d \xi d \tau .
\end{aligned}
$$


Considering Lemma 4, we get $f(x, t) \in F\left(\mathbf{x}_{i}, \mathbf{t}_{j}\right)$ and $k(x, t, \xi, \tau, u(x, t)) \in$ $K\left(\mathbf{x}_{i}, \mathbf{t}_{j}, \mathbf{x}_{l}, \mathbf{t}_{k}, \mathbf{u}_{0}\right)$. Now using (2.3) and the assumptions of the lemma, we replace $x$ and $t$ by $\mathbf{x}_{i}$ and $\mathbf{t}_{j}$ in each subinterval, respectively, to obtain the following interval enclosure

$$
\begin{gathered}
\left.u(x, t)\right|_{x \in \mathbf{x}_{i}, t \in \mathbf{t}_{j}} \in \mathbf{u}_{1, i, j}=F\left(\mathbf{x}_{i}, \mathbf{t}_{j}\right) \\
+\sum_{k=1}^{j-1} \sum_{l=1}^{p_{x}} K\left(\mathbf{x}_{i}, \mathbf{t}_{j}, \mathbf{x}_{l}, \mathbf{t}_{k}, \mathbf{u}_{0}\right)\left(x_{l}-x_{l-1}\right)\left(t_{k}-t_{k-1}\right) \\
+\sum_{l=1}^{p_{x}} K\left(\mathbf{x}_{i}, \mathbf{t}_{j}, \mathbf{x}_{l}, \mathbf{t}_{j}, \mathbf{u}_{0}\right)\left(x_{l}-x_{l-1}\right)\left(\mathbf{t}_{j}-t_{j-1}\right) .
\end{gathered}
$$

Note that

$$
\mathbf{t}_{j}-t_{j-1}=\left[t_{j-1}, t_{j}\right]-t_{j-1}=\left[t_{j-1}-t_{j-1}, t_{j}-t_{j-1}\right]=\left[0, w\left(\mathbf{t}_{j}\right)\right],
$$

so the equation (3.4), can be written as

$$
\begin{aligned}
& \left.u(x, t)\right|_{x \in \mathbf{x}_{i}, t \in \mathbf{t}_{j}} \in \mathbf{u}_{1, i, j}=F\left(\mathbf{x}_{i}, \mathbf{t}_{j}\right) \\
& +\sum_{k=1}^{j-1} \sum_{l=1}^{p_{x}} K\left(\mathbf{x}_{i}, \mathbf{t}_{j}, \mathbf{x}_{l}, \mathbf{t}_{k}, \mathbf{u}_{0}\right) w\left(\mathbf{x}_{l}\right) w\left(\mathbf{t}_{k}\right) \\
& +\sum_{l=1}^{p_{x}} K\left(\mathbf{x}_{i}, \mathbf{t}_{j}, \mathbf{x}_{l}, \mathbf{t}_{j}, \mathbf{u}_{0}\right) w\left(\mathbf{x}_{l}\right)\left[0, w\left(\mathbf{t}_{j}\right)\right] .
\end{aligned}
$$

and this proves the lemma.

In this position, we give an important property of the proposed guaranteed bounds which implies that $\mathbf{u}_{1, i, j}$ is an efficient enclosure in comparison to $\mathbf{u}_{0}$ in each subinterval $\left(\mathbf{x}_{i}, \mathbf{t}_{j}\right)$ :

Theorem 1. Assume that $\max \left\{w\left(\mathbf{x}_{i}\right), w\left(\mathbf{t}_{j}\right)\right\} \leq w\left(\mathbf{u}_{0}\right)$ and there exist $L_{F}, L_{k}$ such that

$$
\left(L_{F}+\chi(b-a) L_{K}\left(1+\frac{\alpha}{p_{t}}\right)\right)<1,
$$

then the new enclosure presented by (3.3) has less width in comparison to initial enclosure $\mathbf{u}_{0}$. 
Proof. Following (2.1), (2.2) and using (3.3), we find that

$$
\begin{gathered}
w\left(\mathbf{u}_{1, i, j}\right) \leq w\left(F\left(\mathbf{x}_{i}, \mathbf{t}_{j}\right)\right) \\
+\sum_{k=1}^{j-1} \sum_{l=1}^{p_{x}} w\left(K\left(\mathbf{x}_{i}, \mathbf{t}_{j}, \mathbf{x}_{l}, \mathbf{t}_{k}, \mathbf{u}_{0}\right)\right) w\left(\mathbf{x}_{l}\right) w\left(\mathbf{t}_{k}\right) \\
+\sum_{l=1}^{p_{x}} w\left(K\left(\mathbf{x}_{i}, \mathbf{t}_{j}, \mathbf{x}_{l}, \mathbf{t}_{j}, \mathbf{u}_{0}\right)\left[0, w\left(\mathbf{t}_{j}\right)\right]\right) w\left(\mathbf{x}_{l}\right) .
\end{gathered}
$$

Now, using (3.1), (3.2) and Lemma 1, we have

$$
\begin{aligned}
w\left(\mathbf{u}_{1, i, j}\right) \leq & L_{F} w\left(\mathbf{u}_{0}\right)+\sum_{k=1}^{j-1} w\left(\mathbf{t}_{k}\right) \sum_{l=1}^{p_{x}} w\left(\mathbf{x}_{l}\right) L_{K} w\left(\mathbf{u}_{0}\right) \\
& +\sum_{l=1}^{p_{x}} L_{K} w\left(\mathbf{x}_{l}\right) \alpha w\left(\mathbf{u}_{0}\right) w\left(\mathbf{t}_{j}\right)
\end{aligned}
$$

and

$$
w\left(\mathbf{u}_{1, i, j}\right) \leq L_{F} w\left(\mathbf{u}_{0}\right)+\chi(b-a) L_{K} w\left(\mathbf{u}_{0}\right)+\chi \frac{(b-a)}{p_{t}} \alpha L_{K} w\left(\mathbf{u}_{0}\right),
$$

so

$$
w\left(\mathbf{u}_{1, i, j}\right) \leq\left(L_{F}+\chi(b-a) L_{K}\left(1+\frac{\alpha}{p_{t}}\right)\right) w\left(\mathbf{u}_{0}\right) .
$$

Finally the condition

$$
\theta=L_{F}+\chi(b-a) L_{K}\left(1+\frac{\alpha}{p_{t}}\right)<1,
$$

gives

$$
w\left(\mathbf{u}_{1, i, j}\right)<w\left(\mathbf{u}_{0}\right)
$$

and the proof is complete.

Remark. We will show later in Section 4, the condition $\theta<1$ in (3.6) is not restrictive in practice and in most cases (3.7) is easily established.

It is well known, in many cases interval initial enclosure may be much wider than the real solution. In order to construct a narrow interval solution, we apply 
Theorem 1 and consider $\mathbf{u}_{1, i, j} \cap \mathbf{u}_{0}$ as a new approximation for $u(x)$. This can be substituted in right side of (3.3) for $\mathbf{u}_{0}$ to give the next approximation and the process is then repeated to achieve a rigorous solution.

Moreover, since the points of subdivision $\left(x_{i}, t_{j}\right)$ for $i=1, \ldots, p_{x}, j=$ $1, \ldots, p_{t}$ interior to $[a, b] \times \Omega$ are common endpoints of boxes $\mathbf{x}_{i} \times \mathbf{t}_{j}, \mathbf{x}_{i} \times$ $\mathbf{t}_{j+1}, \mathbf{x}_{i+1} \times \mathbf{t}_{j}$ and $\mathbf{x}_{i+1} \times \mathbf{t}_{j+1}$, therefore we have

$$
u\left(x_{i}, t_{j}\right) \in u\left(\mathbf{x}_{i}, \mathbf{t}_{j}\right) \subset \mathbf{u}_{1, i, j} \cap \mathbf{u}_{1, i+1, j} \cap \mathbf{u}_{1, i, j+1} \cap \mathbf{u}_{1, i+1, j+1} .
$$

The following algorithm summarizes our strategy where the notations $p_{x}$ and $p_{t}$ are the number of subdivisions in $[0, \chi] \times \Omega$ and $n$ is number of iterations:

Algorithm: Computation of piecewise constant bounds

INPUT:

$$
\begin{aligned}
& n, p_{x}, p_{t} ; \\
& \mathbf{u}_{0} \text { such that } \max \left\{\frac{b-a}{p_{x}}, \frac{\chi}{p_{t}}\right\}<w\left(\mathbf{u}_{0}\right) ;
\end{aligned}
$$

COMPUTE:

$$
\begin{aligned}
& \text { for } k=0, \ldots, n \text { do } \\
& \quad \text { for } i=0, \ldots, p_{x}-1, j=0, \ldots, p_{t}-1 \text { do } \\
& \quad \mathbf{u}_{1, i, j} \text { from }(3.3) ; \\
& \quad \text { if } w\left(\mathbf{u}_{1, i, j} \cap \mathbf{u}_{0}\right) \neq 0 \text { then } \\
& \quad \mathbf{u}_{0}:=\mathbf{u}_{1, i, j} \cap \mathbf{u}_{0} ; \\
& \quad \text { else } \\
& \quad \text { Return: "wrong initial enclosure" } \\
& \quad \text { end-if } \\
& \quad O_{i, j}:=\mathbf{u}_{1, i, j}^{(k)} \cap \mathbf{u}_{1, i+1, j}^{(k)} \cap \mathbf{u}_{1, i, j+1}^{(k)} \cap \mathbf{u}_{1, i+1, j+1}^{(k)} ; \\
& \text { end-for } \\
& \text { end-for } \\
& \text { TPUT: } \\
& O_{i, j}
\end{aligned}
$$

OUTPUT:

Noting that, Theorem 1 shows that the new enclosures are more efficient in comparison to initial bounds, especially when the number of subdivisions is increased. So, in many cases just a few number of iterations is needed in practice and the method rapidly reaches the appropriate solutions. 
The convergence of the proposed algorithm can be characterized by the following theorem:

Theorem 2. Under the hypotheses of Theorem 1, the proposed algorithm is convergent.

Proof. From (3.5), we get

$$
w\left(\mathbf{u}_{1, i, j}\right) \leq\left(L_{F}+\chi(b-a) L_{K}\left(1+\frac{\alpha}{p_{t}}\right)\right) w\left(\mathbf{u}_{0}\right) .
$$

Substituting $\mathbf{u}_{0}$ by $\mathbf{u}_{1, i, j}$ in (3.3) and using Theorem 1 and relation (3.8), the next enclosure $\mathbf{u}_{2, i, j}$ will be obtained as

$$
\begin{aligned}
w\left(\mathbf{u}_{2, i, j}\right) & \leq\left(L_{F}+\chi(b-a) L_{K}\left(1+\frac{\alpha}{p_{t}}\right)\right) w\left(\mathbf{u}_{1, i, j}\right) \\
& \leq\left(L_{F}+\chi(b-a) L_{K}\left(1+\frac{\alpha}{p_{t}}\right)\right)^{2} w\left(\mathbf{u}_{0}\right) .
\end{aligned}
$$

Continuing the process, we get

$$
w\left(\mathbf{u}_{n, i, j}\right) \leq\left(L_{F}+\chi(b-a) L_{K}\left(1+\frac{\alpha}{p_{t}}\right)\right)^{n} w\left(\mathbf{u}_{0}\right) .
$$

Now, according to $\left(L_{F}+\chi(b-a) L_{K}\left(1+\frac{\alpha}{p_{t}}\right)\right)<1$, we conclude

$$
\lim _{n \rightarrow \infty} w\left(\mathbf{u}_{n, i, j}\right)=0,
$$

and this indicates the convergence of interval solutions to a real number in each subinterval.

\section{Numerical experiments and discussions}

Here, we consider two test problems and report the numerical results by the proposed method. Moreover, an adequate discussion on the efficiency of the algorithm is presented. Our discussion includes features and limitations of the scheme e.g. the problem of selecting initial enclosure, the number of iterations and subdivisions. All computations are performed by using the symbolic calculations software Mathematica ${ }^{\circledR}$. 
Example 1. [18] Consider the following nonlinear Volterra-Fredholm integral equation

$$
\begin{aligned}
u(x, t)= & f(x, t)+\int_{0}^{t} \int_{\Omega} G(x, t, \xi, \tau)(1-\exp (-u(u(\xi, \tau)))) d \xi d \tau, \\
& (x, t) \in[0,1] \times \Omega \\
G(x, t, \xi, \tau)= & \frac{x\left(1-\xi^{2}\right)}{(1+t)\left(1+\tau^{2}\right)}, \\
f(x, t)= & -\log \left(1+\frac{x t}{1+t^{2}}\right)+\frac{x t^{2}}{8(1+t)\left(1+t^{2}\right)}
\end{aligned}
$$

where $\Omega=[0,1]$ and the exact solution is

$$
u(x, t)=-\log \left(1+\frac{x t}{1+t^{2}}\right) .
$$

We analyze the problem in three main parts. At first, we compute piecewise bounds on the solution and a comparison is made between the widths of interval solutions and the results of existing classical methods. Then, we show that the conditions of the algorithm and the initial enclosure are not restrictive.

1. Computing piecewise constant bounds: Solution of the equation based on the proposed algorithm for $\mathbf{u}_{0}=[-1,1]$ and $p_{x}=p_{t}=16$ is summarized in Table 1 and Figures 1, 2 for just two iterations. Also, a comparison is made with the numerical results obtained in [18].

\begin{tabular}{|c|c|c|c|c|}
\hline$(x, t)$ & $\begin{array}{c}\text { Exact } \\
\text { solution }\end{array}$ & $\begin{array}{c}\text { Piecewise } \\
\text { bounds }\end{array}$ & $\begin{array}{c}\text { Diam of } \\
\text { enclosures }\end{array}$ & $\begin{array}{c}\text { Results of } \\
{[18]}\end{array}$ \\
\hline$(0.0625,0.0625)$ & -0.00388 & {$[-0.00390,-0.00377]$} & $6.65 \mathrm{E}-5$ & $1.77 \mathrm{E}-5$ \\
$(0.125,0.125)$ & -0.01526 & {$[-0.01545,-0.01477]$} & $3.42 \mathrm{E}-4$ & $5.84 \mathrm{E}-5$ \\
$(0.25,0.25)$ & -0.05715 & {$[-0.05932,-0.05500]$} & $2.15 \mathrm{E}-3$ & $-5.39 \mathrm{E}-4$ \\
$(0.5,0.5)$ & -0.18232 & {$[-0.23766,-0.16935]$} & $3.41 \mathrm{E}-2$ & $-1.22 \mathrm{E}-2$ \\
\hline
\end{tabular}

Table 1 - Numerical results of example 4.1 for $n=2$ and $p_{x}=p_{t}=16$.

The reported results in Table 1 show that since these enclosures include all round-off errors in the computation, interval methods are valuable tools in comparison to classical schemes. Figure 1 indicates that increasing the number of subdivisions led to better numerical results. 

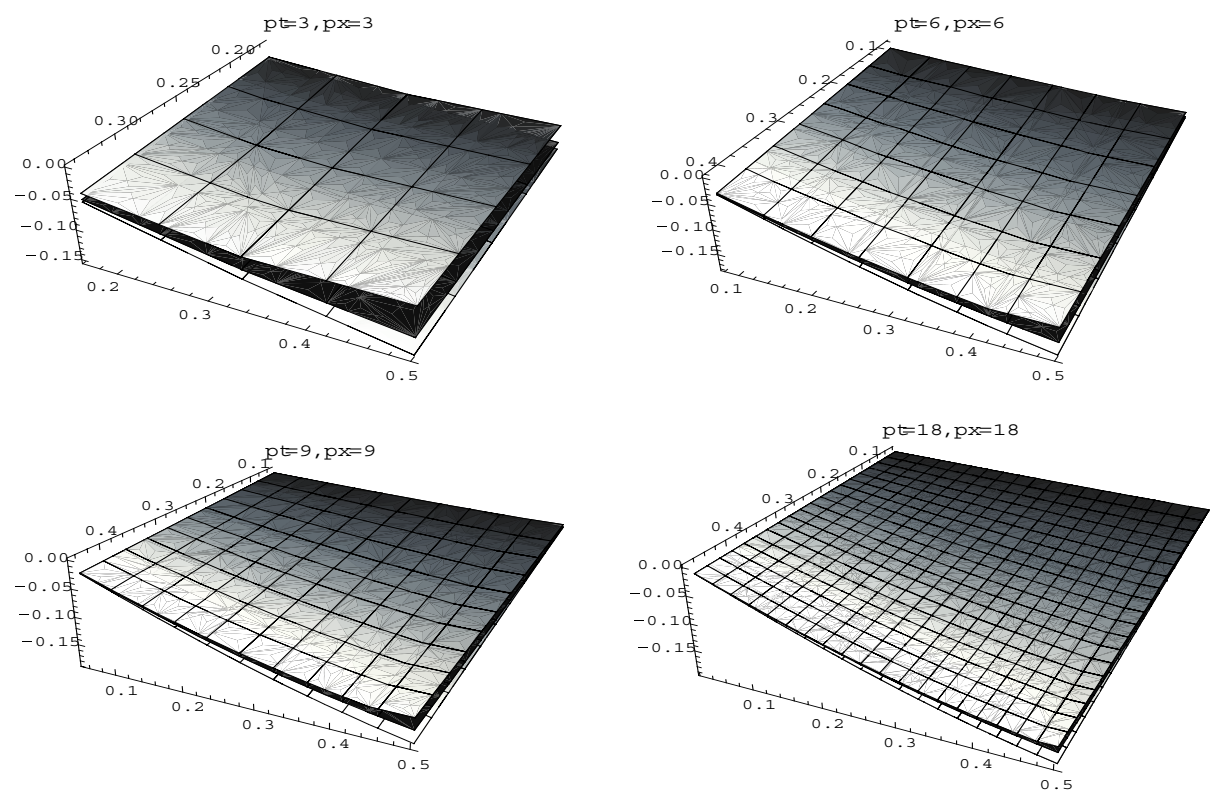

Figure 1 - Exact solution of example 4-1 (black sheet) is enclosed in piecewise constant bounds.
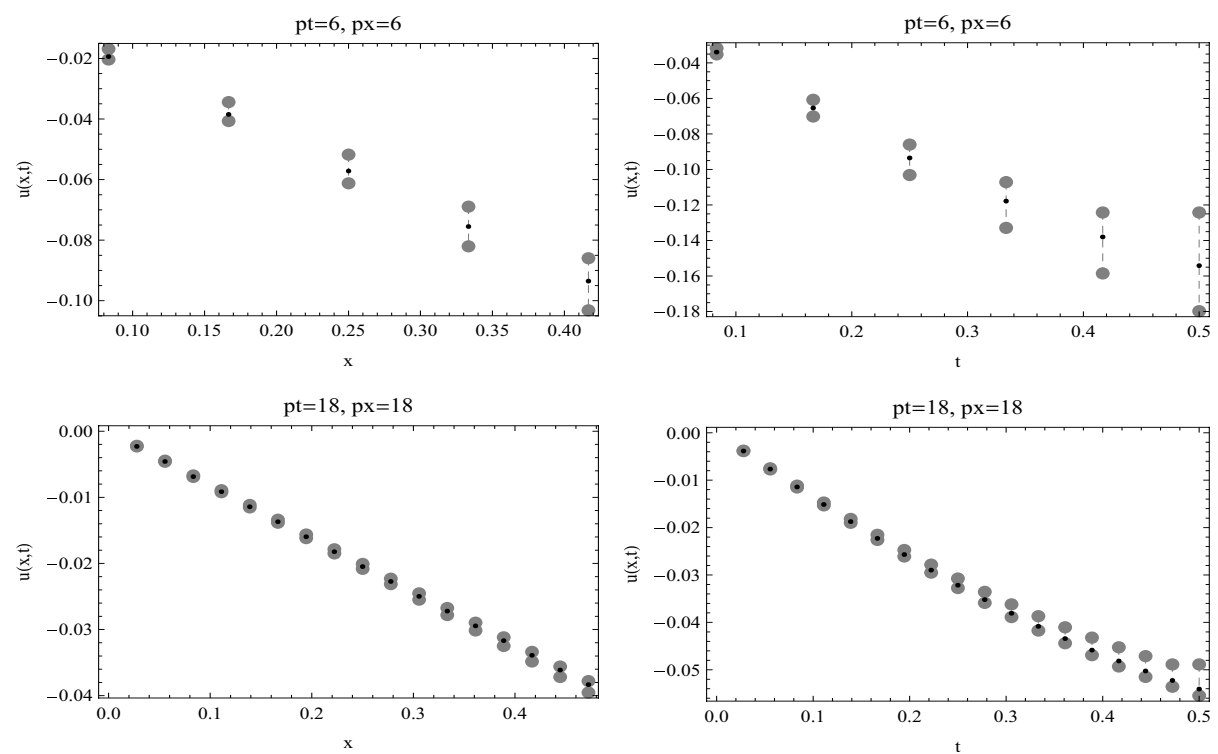

Figure 2 - Exact solution of example 4-1 (black dots) is enclosed in piecewise constant bounds (Gray circles). 


\section{Discussion on the convergence conditions}

The following table gives an approximation for $L_{k}$ and $L_{F}$ in (3.1) and (3.2):

\begin{tabular}{|c|c|c|c|c|c|c|}
\hline$(\mathbf{x}, \mathbf{t})$ & $\mathbf{u}_{0}$ & $w\left(K\left(\mathbf{u}_{0}\right)\right)$ & $L_{F}$ & $L_{K}$ & $\alpha$ & $\begin{array}{c}\theta=L_{F}+\chi(b-a) \\
L_{K}\left(1+\frac{\alpha}{p_{t}}\right)\end{array}$ \\
\hline$([0.0,0.1],[0.0,0.1])$ & {$[-0.1,0.1]$} & 0.020 & 0.10 & 0.10 & 0.52 & 0.20 \\
$([0.3,0.4],[0.2,0.3])$ & {$[-0.1,0.1]$} & 0.040 & 0.58 & 0.20 & 0.52 & 0.79 \\
$([0.2,0.3],[0.1,0.2])$ & {$[-1.0,1.0]$} & 0.037 & 0.39 & 0.18 & 0.73 & 0.58 \\
$([0.4,0.5],[0.4,0.5])$ & {$[-0.5,0.5]$} & 0.260 & 0.66 & 0.260 & 0.62 & 0.87 \\
\hline
\end{tabular}

Table 2 - Approximating Lipshitz constants and $\theta$ in (3.6).

Analyzing the results of Table 2 shows that the conditions (3.7) and (3.9) are easily established. In some cases, due to the dependency effect the width of $F$ and $K$ may be wider than usual, so as previously mentioned, we can use mean value form or other Taylor enclosures for $F$ and $K$. For example in Table 2, $L_{F}=0.66$ for $([0.4,0.5],[0.4,0.5])$ is obtained by using the mean value form of $F$. (For more detail see e.g. [20]).

\section{Discussion on $u_{0}$ and number of iterations}

The problem of choosing initial enclosure $\mathbf{u}_{0}$ seems to be another restrictive difficulty. Table 3 illustrates the piecewise bounds obtained by choosing various initial enclosures and number of iterations:

\begin{tabular}{|c|cc|cc|cc|}
\hline \multirow{2}{*}{$(x, t)$} & \multicolumn{2}{|c|}{$\mathbf{u}_{0}=[-2,2]$} & \multicolumn{2}{c|}{$\mathbf{u}_{0}=[2,3]$} & \multicolumn{2}{c|}{$\mathbf{u}_{0}=[-5,5]$} \\
\cline { 2 - 7 } & $n=2$ & $n=10$ & $n=2$ & $n=5$ & $n=2$ & $n=10$ \\
\hline$(0.0625,0.0625)$ & $7.44 \mathrm{E}-5$ & $6.19 \mathrm{E}-5$ & 0 & 0 & $3.23 \mathrm{E}-4$ & $6.19 \mathrm{E}-5$ \\
$(0.125,0.125)$ & $4.55 \mathrm{E}-4$ & $2.21 \mathrm{E}-4$ & 0 & 0 & $5.78 \mathrm{E}-3$ & $2.85 \mathrm{E}-4$ \\
$(0.250,0.25)$ & $3.71 \mathrm{E}-3$ & $1.45 \mathrm{E}-3$ & 0 & 0 & $3.72 \mathrm{E}-2$ & $1.45 \mathrm{E}-3$ \\
$(0.5,0.5)$ & $5.50 \mathrm{E}-2$ & $9.47 \mathrm{E}-3$ & 0 & 0 & $2.41 \mathrm{E} 0$ & $5.84 \mathrm{E}-1$ \\
\hline
\end{tabular}

Table 3 - Diameter of solutions of example 4.1 for $p_{x}=p_{t}=16$.

Our experimental results in Table 3, show:

(i) If the computed interval is empty (as for $\mathbf{u}_{0}=[2,3]$ ), it implies that we choose an inaccurate $\mathbf{u}_{0}$ which does not contain exact solution. 
(ii) If we choose a proper $\mathbf{u}_{0}$ just a few iteration is needed to achieve rigorous solutions, moreover increasing the number of iterations can offset inappropriate $\mathbf{u}_{0}$.

Example 2. [14]

$$
\begin{aligned}
u(x, t)= & f(x, t)+\int_{0}^{t} \int_{\Omega} G(x, t, \xi, \tau) \sin (u(\xi, \tau)) d \xi d \tau, \\
& (x, t) \in[0,1] \times \Omega, \\
G(x, t, \xi, \tau)= & x t(\xi+\tau), \\
f(x, t)= & -t\left(x^{2}-\sin (x)-x \sin (x)\right),
\end{aligned}
$$

where $\Omega=[0,1]$ and $u(x, t)=x t$.

Solution of the equation using the proposed scheme is enclosed in intervals as we show in Table 4 and Figures 3, 4. The following experiments are also observed:

(i) Similar to the previous example, for an inaccurate $\mathbf{u}_{0}=[-2,-1]$ the computed interval is empty.

(ii) In many cases, increase the number of iterations does not affect the accuracy of the results, therefore to increase the accuracy we need to increase the number of subdivisions (see e.g. Figure 4).

\begin{tabular}{|c|cc|cc|}
\hline \multirow{2}{*}{$(x, t)$} & \multicolumn{2}{|c|}{$\mathbf{u}_{0}=[0,1]$} & \multicolumn{2}{c|}{$\mathbf{u}_{0}=[-2,-1]$} \\
\cline { 2 - 5 } & $n=2$ & $n=10$ & $n=2$ & $n=5$ \\
\hline$(0.0625,0.0625)$ & $2.44 \mathrm{E}-4$ & $2.44 \mathrm{E}-4$ & 0 & 0 \\
$(0.125,0.125)$ & $9.77 \mathrm{E}-4$ & $9.77 \mathrm{E}-4$ & 0 & 0 \\
$(0.250,0.25)$ & $3.94 \mathrm{E}-3$ & $3.94 \mathrm{E}-3$ & 0 & 0 \\
$(0.5,0.5)$ & $2.24 \mathrm{E}-2$ & $2.24 \mathrm{E}-2$ & 0 & 0 \\
\hline
\end{tabular}

Table 4 - Diameter of solutions of example 4.2 for $p_{x}=p_{t}=16$.

\section{Conclusion}

Nonlinear Volterra-Fredholm integral equations have many applications in science and engineering, but in many cases, accumulation of truncation and roundoff errors prevent the numerical methods to converge to the exact solution. In this 

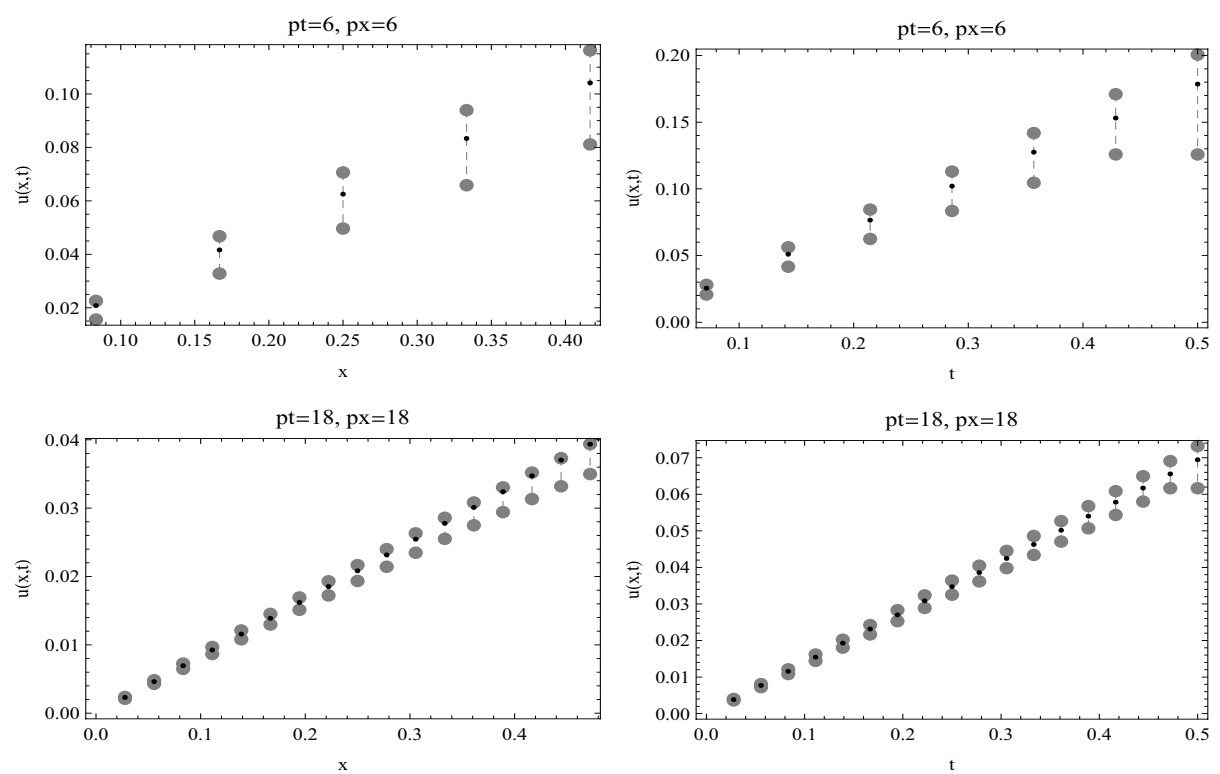

Figure 3 - Exact solution of example 4-2 (black dots) is enclosed in piecewise constant bounds (gray circles).
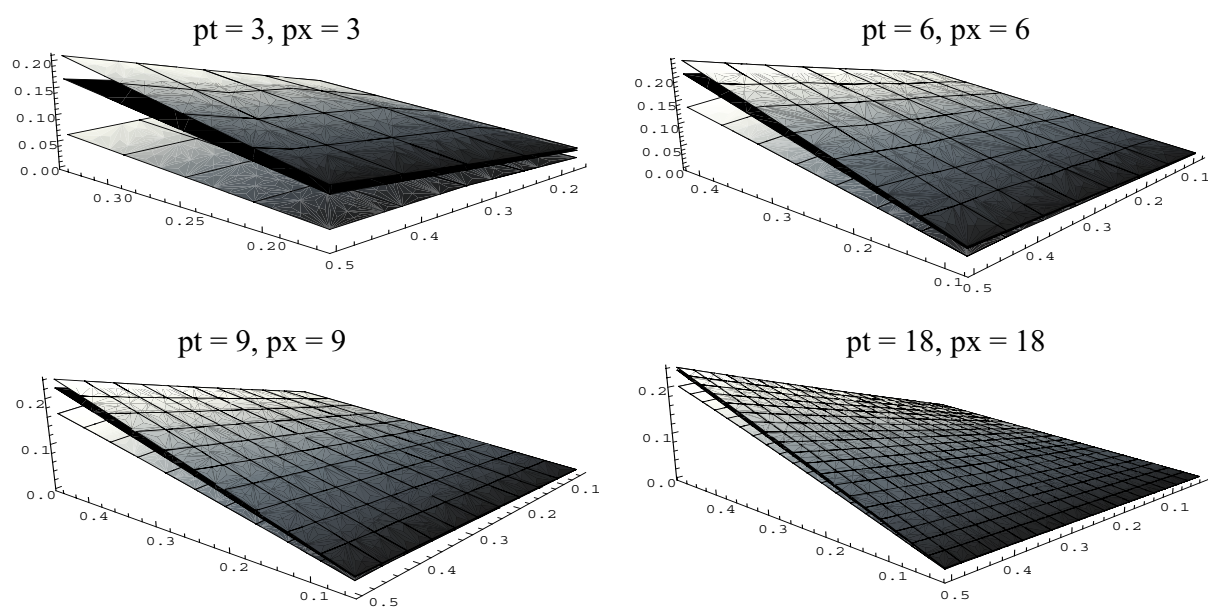

Figure 4 - Exact solution of example 4-2 (black sheet) is enclosed in piecewise constant bounds. 
paper, we consider an initial enclosure and obtain a new guaranteed bounds for the solution of these equations including all round-off and truncation errors. We also clarify that the algorithm initial enclosures and conditions are not restrictive in practice. Our numerical experiments show that the exact solution is enclosed in intervals where the width of intervals gives the accuracy of the numerical results. This is of great importance, especially when the equations are complicated and the solution is not determined analytically or numerically.

\section{REFERENCES}

[1] G. Alefeld and G. Mayer, Interval analysis: theory and applications. J. Comput. Appl. Math., 121 (2000), 421-464.

[2] H. Brunner, On the numerical solution of nonlinear Volterra-Fredholm integral equation by collocation methods. SIAM J. Numer. Anal., 27 (1990), 987-1000.

[3] H. Brunner and E. Messina, Time-stepping methods for Volterra-Fredholm integral equations by collocation methods. Rend. Math., 23 (2004), 329-342.

[4] T.A. Burton, Volterra Integral and Differential Equations. Academic Press, New York (1983).

[5] O. Caprani, K. Madsen and O. Stauning, Enclosing solutions of integral equations. Technical Report, Tech. Univ. of Denmark (1996).

[6] M.B. Dhakne and S.D. Kendre, On abstrct nonlinear mixed Volterra-Fredhom integro-differential equations. Comm. Appl. Nonlinear Anal., 4 (2006), 101-112.

[7] O. Diekmann, Thresholds and travelling waves for the geographical spread of infection. J. Math. Biol., 6 (1978), 109-130.

[8] H.J. Dobner, Computing narrow inclusions for the solution of integral equations. Numer. Func. Anal. Optim., 10 (1989), 923-936.

[9] H.J. Dobner, A method for estimating the solution of integral equations encountered in potential theory. Appl. Math. Comput., 109 (2000), 199-204.

[10] K. Domke and L. Hacia, Integral equation in some thermal problem. Int. J. Math. Comput. Simulation, 2 (2007), 184-189.

[11] W. Edmonson, S. Ocloo, C. Williams and W. Alexander, The use of interval methods in signal processing and control for systems biology edmonson, Foundations of Computational Intelligence, IEEE Symposium (2007), 136-142.

[12] J.W.V. Gudenberg, Interval arithmetic on multimedia architectures. Reliab. Comput., 8 (2002), 307-312. 
[13] H. Guoqiang, Asymptotic error expansion for the Nystrom method for a nonlinear Volterra-Fredholm equations. J. Comput. Appl. Math., 59 (1995).

[14] M. Hadizadeh and Gh. Kazemi, Error estimate in the Sinc collocation method for the Volterra-Fredhlom integral equations based on DE Transformation. Elect. Trans. Numer. Anal., 30 (2008), 75-87.

[15] M. Hadizadeh and S. Yazdani, New enclosure algorithms for the verified solutions of nonlinear Volterra integral equations. Appl. Math. Modelling, 35 (2011), 29722980.

[16] J.P. Kauthen, Continuous time collocation methods for Volterra-Fredholm integral equations. Numer. Math., 56 (1989), 409-424.

[17] J.L. Lions, Ariane 5, flight 501 failure. Technical report, European Space Agency (1996).

[18] K. Maleknejad and M. Hadizadeh, A new computational method for VolterraFredholm integral equations. Computer Math. Applic., 37 (1999), 1-8.

[19] R.E. Moore, Interval Analysis. Prentice-Hall, New York (1966).

[20] R.E. Moore, R.B. Kearfott and M.J. Cloud, Introduction to Interval Analysis. SIAM (2009).

[21] S. Murashige and S. Oishi, Numerical verification of solutions of periodic integral equations with a singular kernel. Numer. Algor., 37 (2004), 301-310.

[22] R. Muhanna, H. Zhang and R. Mullen, Interval finite elements as a basis for generalized models of uncertainty in engineering mechanics. Reliab. Comput., 13 (2007), 173-194.

[23] M. Neher, K.R. Jackson and N.S. Nedialkov, On Taylor model based integration of ODES. SIAM J. Numer. Anal., 45 (2007), 236-262.

[24] H.R. Thieme, A model for the spatial spread of an epidemic, 4 (1977), 337-351.

[25] H.L. Tidke, Existence of global solutions to nonlinear mixed Volterra-Fredholm integro-differential equations with nonlocal conditions. Electron. J. Diff. Equa., 55 (2009), 1-7. 